\title{
Biological Behavior and Lipid Metabolism of Colon Cancer Cells are Regulated by a Combination of Sterol Regulatory Element-Binding Protein I and ATP Citrate Lyase
}

This article was published in the following Dove Press journal: OncoTargets and Therapy

\author{
Zhendong Qiu* \\ Wenhong Deng* \\ Yupu Hong \\ Liang Zhao \\ Man Li \\ Yongjun Guan \\ Yingru Su \\ Chen Chen \\ Qiao Shi \\ Jia Yu \\ Weixing Wang (D)
}

Department of General Surgery, Renmin Hospital of Wuhan University, Wuhan, 430060, Hubei Province, People's

Republic of China

*These authors contributed equally to this work

\begin{abstract}
Purpose: To research the effects of ATP citrate lyase (ACLY) and Sterol-regulatory element binding protein 1 (SREBP1) on the biology and lipid metabolism of colorectal cancer cells. Methods: Colorectal cancer cells Caco-2 and Lovo were transfected with ACLY or SREBPI gene knockdown lentiviruses. Four groups were set: $A C L Y$ knockdown, SREBP1 knockdown group, empty vector-transfected (negative control), and untreated cells (blank control). Cell proliferation was measured using CCK-8, colony formation, and EdU labeling assays. Apoptosis was detected using Annexin V-APC/7- AAD and JC-1 assay. Transwell migration and wound healing assays analyzed cell migration and invasion. A triglyceride test kit and oil red $\mathrm{O}$ stain assessed cell lipid production. Key factors related to lipid metabolism were detected.

Results: $A C L Y$ and SREBP1 promoted cell proliferation at 48 and $120 \mathrm{~h}$, but there was no significant difference in Caco-2 cells at $24 \mathrm{~h}$, at which point the effect of SREBP1 was more important. $A C L Y$ 's effect on cell proliferation was more obvious at $120 \mathrm{~h}$. Colony formation assays in Caco-2 showed similar results to the CCK-8 assay at $120 \mathrm{~h}$, but $A C L Y$ knockdown had no effect in Lovo cells. EDU assays showed that $A C L Y$ or $S R E B P 1$ facilitated DNA reproduction in the two cell lines, in which SREBP1 was more significant. Knockdown of the two genes showed significant differences in Lovo cells. However, $A L C Y$ knockdown promoted apoptosis to a greater extent than SREBP1 knockdown in Caco-2 cells. In addition, ACLY and SREBP1 enhanced migration, invasion, and lipid production in both cell lines. Knockdown of $A C L Y$ or $S R E B P 1$ reduced lipid metabolism pathway gene expression in the two cell lines.
\end{abstract}

Conclusion: Knockdown of $A C L Y$ and SREBP1 genes inhibit the proliferation, migration, and invasion of colorectal cancer cells, while promoting their apoptosis. Our results identified potential new targets to treat colorectal cancer via lipid synthesis modulation in cancer cells.

Keywords: ATP-citrate lyase, colorectal cancer, lipid metabolism, sterol-regulatory element binding protein 1

\section{Introduction}

Colorectal cancer (CRC) is one of the most common malignancies worldwide. It is the second leading cause of cancer death, thus there is an urgent need to reduce the incidence and mortality rate using innovative strategies to improve prevention, early diagnosis, prognostic biomarkers, and treatment effectiveness. ${ }^{1}$ In recent years, glucose metabolism of cancer has attracted research attention. However, the involvement of fatty acid metabolism is not so well studied.
Correspondence: Weixing Wang; Jia Yu Department of General Surgery, Renmin Hospital of Wuhan University, 238 Jiefang Road, Wuhan, 430060, Hubei Province,

People's Republic of China Email sate.llite@I63.com; yogaqqI16@163.com 
In normal cell metabolism, fatty acids are derived from two sources. Diet provides exogenous fatty acids, while endogenous fatty acids are produced through de novo lipogenesis (DNL). ${ }^{2}$ In cancer cells, $93 \%$ of fatty acid are derived from DNL, and DNL-related enzymes are overexpressed. ${ }^{3}$ One of the crucial steps in the process of colorectal cancer distant metastasis is epithelial-mesenchymal transition (EMT), which is closely related to lipid metabolism and enables the formation of migratory mesenchymal cells with an invasive phenotype. Loss of cellular adhesion proteins (such as E-cadherin), tight junction proteins, and the concomitant expression of mesenchymal markers are generally accepted as hallmarks of EMT. In addition, EMT is associated with a poor prognosis of patients with tumors. ${ }^{4}$

The rapid proliferation of tumor cells requires a sufficient supply of lipids, including phospholipid and cholesterol. ${ }^{5}$ Moreover, lipids constitute the main structural fatty acids and glycerides of biological plasma membrane to provide potential energy substrates, which support the rapid proliferation of the tumor. ${ }^{6}$ In addition, lipids are the second messengers of DNL, mediating signal transduction pathways that are vital for tumor antiangiogenic therapy. ${ }^{7}$ Once treatment is stopped, tumor cells consume a considerable amount of NADPH to obtain sufficient energy to resume the ab initio synthesis of fatty acids. ${ }^{8}$

The sterol regulatory element-binding proteins (SREBPs) are transcription factors that can regulate the key enzymes in the synthesis of cholesterol, fatty acids, triglycerides and phospholipids. ${ }^{9}$ SREBPs comprise SREBP1a, SREBP1c, and SREBP2. SREBP2 mainly regulates cholesterol metabolism, while SREBP1 can simultaneously stimulate the expression of fatty acids and cholesterol-related enzymes. ${ }^{10,11}$ After the reaction of a series of substrate enzymes: ATP Citrate lyase (ACLY), fatty acid synthase (FASN), acetyl-CoA carboxylase (ACC) and stearoyl-CoA desaturase 1 (SCD1), acetylcoenzyme A (AC-CoA) synthesizes palmitate products, which enter the final pathway of fatty acid synthesis. Among these enzymes, ACLY is a key upstream enzyme in DNL, whose gene expression is abnormally high in a variety of malignancies. ${ }^{12}$ It functions as a tetramer, and is composed of citryl-CoA synthetase (CCS) $\beta$ and CCS $\alpha$ regions, as well as an N-terminal CCS module. ${ }^{13}$ ACLY is mainly located in the endoplasmic reticulum, but can also be detected in the nucleus of certain types of cells, such as mouse embryonic fibroblasts, mouse pre-B lymphocytes, human glioblastoma, and colorectal cancer cells. ${ }^{14}$ Inhibition of ACLY can delay the occurrence of tumors. ${ }^{15,16}$ To date, a large number of studies have been carried out to confirm the importance of ACLY in alcoholic fatty liver, coronary atherosclerosis, tumors, and other diseases. For example, a previous study showed that the consumption of fish oil reduced blood lipids and restrained the activity of ACLY by downregulating SREBP1 in humans. ${ }^{17}$ However, the roles of SREBPs in lipid metabolism of colorectal cancer cells remain unclear. This present study aimed to explore the role of the ACLYSREBP1 pathway in colorectal cancer. Here, we showed that the ACLY-SREBP1 axis enhances lipid synthesis, facilitates the proliferation, migration, and invasion of CRC cell lines, and inhibits their apoptosis, which suggested that inhibition of the ACLY/SREBP1 axis could be exploited as a novel target for metabolic therapy in CRC.

\section{Materials and Methods Cell Lines and Reagents}

CRC cell lines Caco-2 and Lovo were obtained from the Kunming Cell Bank, Chinese Academy of Sciences, and were maintained in Dulbecco's modified Eagle's medium (DMEM) high glucose medium (GinoBio, China, GNM12800) supplemented with $10 \%$ fetal bovine serum (FBS)(Gibco, South American, 10270-106), and $100 \mathrm{U} / \mathrm{mL}$ penicillin and $100 \mu \mathrm{g} / \mathrm{mL}$ streptomycin (HyClone, Logan, UT, USA, SH4003.01).

\section{Generation of Stable Cell Lines}

To establish stable $A C L Y$ and $S R E B P 1$ knockdown cell lines, cells were transfected with commercially available lentiviruses (Genechem, Shanghai, China) that express $A C L Y$ targeted short hairpin RNA (shRNA) (termed LV-ACLYRNAi), SREBP1-targeted shRNA (termed LV-SREBP1RNAi), or control shRNA. The plasmids are based on a lentiviral vector with puromycin selection and a green fluorescent protein (GFP) cassette. Cells were transduced with the lentiviral vector using a multiplicity of infection (MOI) of 10 and $10 \mu \mathrm{g} / \mathrm{mL}$ HitransG for $48 \mathrm{~h}$. The transfected cells were then maintained in 6-well plates and purified with $3 \mu \mathrm{g} / \mathrm{mL}$ (Lovo) and $5 \mu \mathrm{g} / \mathrm{mL}$ (Caco-2) of puromycin, respectively, for 1 week prior to experiments. The transfection rate was verified by fluorescence imaging, flow cytometry, and Western blotting. To facilitate subsequent experiments, another batch of lentiviruses without GFP labeling were used for partial assays. Each cell line was divided into four groups according to cell treatment: 
An $A C L Y$ knockdown group, an SREBP1 knockdown group, cells transfected with empty vector as the negative control (NC) group, and untreated cells as a blank control group.

\section{Western Blotting Assay}

Cells were cultured under specific conditions, and total protein was extracted using Radioimmunoprecipitation assay (RIPA) lysis buffer (Beyotime, Shanghai, China). Equal amounts of proteins were separated using sodium dodecyl sulfate polyacrylamide gel electrophoresis (SDS-PAGE) and transferred to a polyvinylidene fluoride (PVDF) membrane (Millipore, Darmstadt, Germany). After blocking with 5\% non-fat milk for $2 \mathrm{~h}$ at room temperature, the membrane was incubated with antibodies recognizing hydroxy methylglutaryl CoA reductases (HMGCR) (sc-271595, Santa Cruz Biotechnology, Santa Cruz, CA, USA), acetyl-CoA carboxylase 1 (ACC1) (21923-1-AP, Proteintech, Chicago, IL, USA), SREBP1 (ab28481, Abcam, Cambridge, MA, USA), ACLY (ab40793, Abcam), glyceraldehyde-3-phosphate dehydrogenase (GAPDH) (ab9485, Abcam), and lowdensity lipoprotein receptor (LDLR) (ab189170, Abcam) at $4^{\circ} \mathrm{C}$ overnight. The membranes were then incubated with the appropriate secondary antibodies at room temperature for 2 h. At the end of the incubation, an enhanced chemiluminescence kit (Biosharp, Hefei, China) was used to detect the immunoreactive protein bands, and images were captured using the ChemiDoc XRS imaging system (Bio-Rad Laboratories, Hercules, CA, USA). Goat anti-rabbit (GB23303) and goat anti-mouse (GB23302) secondary antibodies were purchased from Servicebio (Wuhan, China).

\section{Cell Viability Assay}

Cells were plated in 96-well plates at a density of $5 \times 10^{3}$ cells per well and treated with complete medium (containing $10 \% \mathrm{FBS}$ ) for 24,48 , and $72 \mathrm{~h}$. At the indicated time points, a Cell Counting Kit-8 (CCK-8, Dojindo, Shanghai, China, CK04) was used to detect cell viability at $37^{\circ} \mathrm{C}$ for $1 \mathrm{~h}$. The luminescence of each sample was determined at $450 \mathrm{~nm}$ using a microplate reader (PerkinElmer, Waltham, MA, USA). The percentage of viable cells was estimated in comparison with the untreated controls. At least three independent experiments were performed.

Next, $2 \times 10^{4}$ cells were seeded in 12-well plates, washed with phosphate-buffered saline (PBS) after $24 \mathrm{~h}$, fixed using 4\% paraformaldehyde for $15 \mathrm{~min}$, followed by incubation with $0.1 \%$ crystal violet solution and observed under a microscope.
Cells in the logarithmic growth phase were digested and re-suspended in PBS in a 12-well plate. Next, 1\% 5-Ethynyl-2'-deoxyuridine (EdU) (Beyotime, Shanghai, China, C0078S) working solution was added to each well $24 \mathrm{~h}$ later and incubated at $37^{\circ} \mathrm{C}$ for $2 \mathrm{~h}$. The working solution was then removed, and the cells were fixed in $4 \%$ paraformaldehyde for 30 minutes, before being incubated for $15 \mathrm{~min}$ at room temperature in a permeability solution containing $0.3 \%$ Triton-X-100. Then, according to the EdU kit instructions, the click working liquid was added and incubated for $30 \mathrm{~min}$ at room temperature in the dark. Finally, the film was sealed with 4',6-diamidino-2-phenylindole (DAPI) (ab104139, Abcam, Cambridge, UK) and photographed under an inverted fluorescence microscope (MicroPublisher 5.0 RTV, QIMAGING, Canada).

\section{Colony Formation Assay}

Cells were diluted to the appropriate density, and 500 cells per well were seeded in 12-well plates. After culture for 2 weeks, the cells were fixed with $4 \%$ paraformaldehyde and stained with $0.1 \%$ crystal violet solution. The number of colonies was counted using the ImageJ software (NIH, Bethesda, MA, USA).

\section{Lipid Accumulation}

Cells grown on glass coverslips were fixed in 4\% paraformaldehyde (MKCB4217, Sigma, St. Louis, MO, USA) for $20 \mathrm{~min}$, and rinsed with $60 \%$ freezing isopropanol for 5 min. Then, the cells on the coverslips were stained for 30 min at room temperature with freshly prepared Oil Red O working solution (Solarbio, Beijing, China, cat: G1260), re-stained with Hematoxylin (Servicebio, cat: G1004) and then rinsed with water. The sections were observed under a phase-contrast microscope (Olympus, Tokyo, Japan) and IOD value of Oil Red $\mathrm{O}$ was calculated with Image-Pro Plus.

Triglycerides (TGs) in cells were measured using commercial kits (Jiancheng Technology Co., Nanjing, China) according to the manufacturer's instructions. The luminescence of each sample was determined at $450 \mathrm{~nm}$ using a microplate reader (PerkinElmer). The TG content $=[($ sample value - blank value)/(calibration value - blank value)] $\times$ calibration product concentration/sample protein concentration to be tested. Each sample is tested in three wells.

\section{Apoptosis Assay}

After culture under the indicated conditions, cells were collected and stained with an Annexin V- Allophycocyanin 
(APC)/7-Aminoactinomycin D (7- AAD) apoptosis detection kit (BD, 559763). The percentage of apoptotic cells was detected by flow cytometry (FACSCalibur, BD Biosciences, San Jose, CA, USA). Annexin V-APC+ staining represented early apoptotic cells, and 7-AAD+ staining represented late apoptotic cells. For each group, 15,000 cells were analyzed. At least three independent experiments were performed.

Cells in logarithmic growth phase were then digested using trypsin in EDTA and seeded in 12-well plates. Twenty four hours later, the culture medium was discarded. Then, $1 \mathrm{~mL}$ of a JC-1 (Beyotime, C2006) working solution was added to each well and incubated at $37^{\circ} \mathrm{C}$ for 20 minutes. The cells were washed twice with JC-1 Buffer, and photographed under an inverted fluorescence microscope.

\section{Transwell Invasion Assay}

Transwell migration assays were completed on $8-\mu \mathrm{m}$ pore membranes in a 24 -well format. $2 \times 10^{4}$ cells in DMEM high glucose medium were seeded in the upper chamber and FBS was added only onto the lower chamber as a chemoattractant. After $24 \mathrm{~h}$, cells that did not migrate were cleaned off the top of the membrane using a cotton swab, and migrated cells were fixed, stained with crystal violet, and mounted onto slides. The average value of at least three visual fields was taken, and the experiment was performed three times.

\section{Wound Healing Assay}

In addition, a wound-healing assay was used to determine cell migration. About $5 \times 10^{5}$ cells were seeded into a 6-well plate. When the cells grew to near confluence, a wound was made in the monolayer cells using a $10 \mu \mathrm{L}$ pipette tip. After the indicated treatment, the wounds were imaged at 0,12 , and $24 \mathrm{~h}$ and the difference was calculated using the ImageJ software.

\section{Statistical Analysis}

Statistical analyses were performed using the SPSS software package (SPSS26.0; IBM Corp., Armonk, NY, USA). Statistical analyses for multiple groups were performed using one_way ANOVA followed by the least significant difference (LSD) post hoc test. $\mathrm{P}<0.05$ was considered statistically significant. Each experiment was performed at least three times.

\section{Results}

\section{Knockdown of ACLY and SREBPI} Inhibited the Proliferation of Colorectal

\section{Cancer Cells}

Initially, the levels of ACLY and SREBP1 in NCM460, Caco-2, Lovo, HCT116, and SW480 cells were compared using Western blotting (Figure 1A and B). ACLY and SREBP1 levels were upregulated in Caco-2 and Lovo cell lines. To understand the potential role of ACLY or SREBP1 in CRC cells, ACLY-specific and SREBP1specific shRNAs were used, and ACLY and SREBP1 were detected using fluorescence microscope and Western blotting analysis. The $A C L Y$-specific and SREBP1-specific shRNAs effectively knocked down $A C L Y$ and SREBP1 expression in Caco-2 and Lovo cells (Figure 1C-F).

$A C L Y$ and SREBP1 knockdown CRC cells were incubated in 96-well plates for 24, 48, and $120 \mathrm{~h}$, followed by a CCK-8 assay (Figure 2C). Knockdown of ACLY and $S R E B P 1$ generally promoted cell proliferation, but there were some differences among the groups. Knockdown of $A C L Y$ or $S R E B P 1$ decreased cell proliferation at 48 and 120 $\mathrm{h}$, in which knockdown of SREBP1 caused a larger decrease in cell proliferation than knockdown of $A C L Y$. Furthermore, the difference in cell proliferation caused by $A C L Y$ knockdown was more obvious at $120 \mathrm{~h}$ among the three time points. Next, we assessed whether knockdown of $A C L Y$ or SREBP1 also inhibited the colony formation capacity of CRC cells (Figure 2A and B). In the colony formation assay of Caco-2 cells, the number of clones per plate decreased significantly in the knockdown group, especially in the SREBPI knockdown group. In Lovo cells, there was no significant difference in the number of clones per plate between $A C L Y$ knockdown group and the control group, but the number of clones per plate in the SREBP1 knockdown group decreased sharply. In the EdU proliferation test (Figure 2D-F), the results were similar to those in the CCK-8 assay at $120 \mathrm{~h}$, but slightly different from those in CCK-8 at $24 \mathrm{~h}$. The amount of EdU in Lovo cells after $A C L Y$ knockdown was significantly lower than that in the control group, which indicated that the total proliferation ability of Lovo cells remained unchanged; however, their DNA replication ability decreased. The above proliferation tests revealed the differences between early and relatively late cell proliferation of CRC cells, and suggested the possible mechanism of early proliferation. 
A

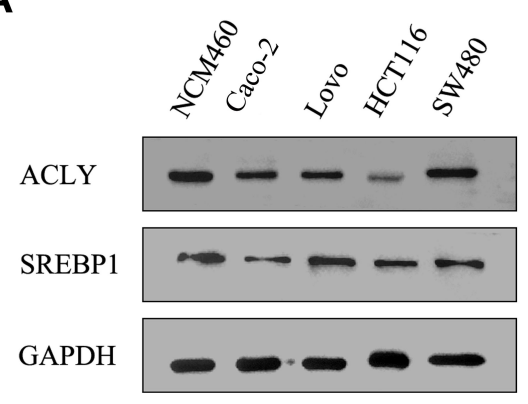

B

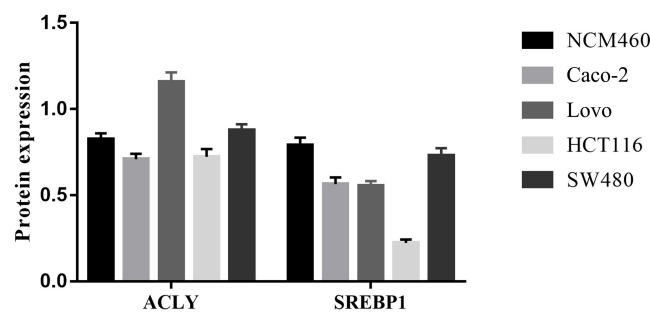

C ACLY
GAPDH

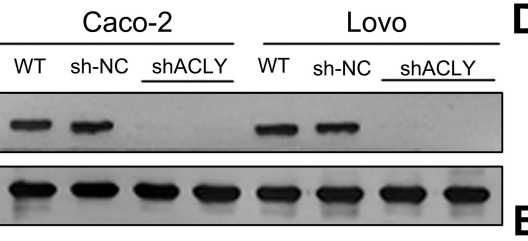

E

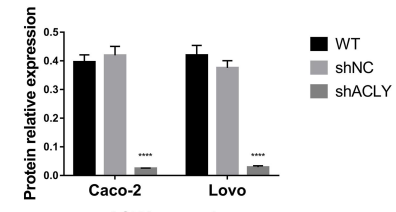

WT sh-NC shSREBP1 WT sh-NC ShSREBP1

SREBP

GAPDH
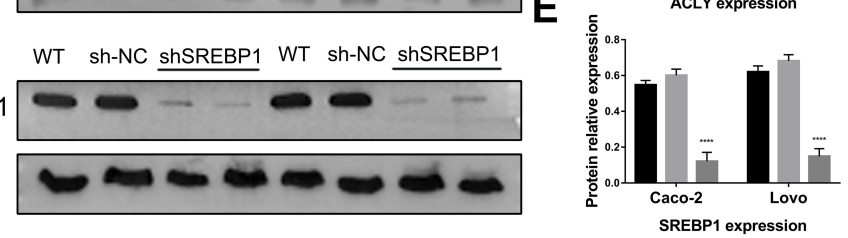

WT shSREBP1

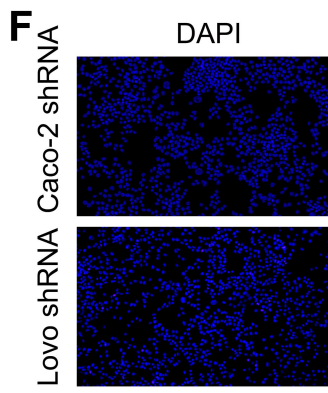

GFP

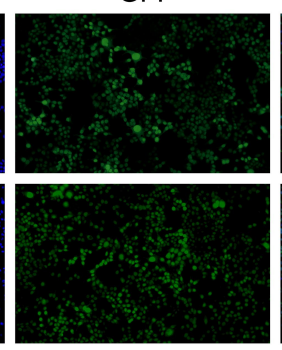

Merge

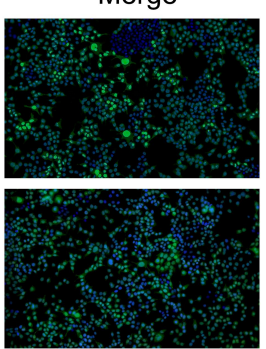

Figure I The knockout effect of ACLY or SREBPI in Caco-2 and Lovo. (A and B) ACLY and SREBPI expression of NCM460, Caco-2, Lovo, HCTII6 and SW480. (C-E) The effects of knockout on the ACLY and SREBPI in Caco-2 and Lovo were detected by Western blotting. GAPDH was used as an internal loading control. (F) After knockout treatment, immunofluorescence staining was performed to evaluate the green fluorescent protein (GFP) expression levels in Caco-2 and Lovo cells. GFP staining is shown in green, and nuclear staining is shown in blue. The magnification is $100 \times$. $* * * * P<0.0001$ vs control group.

\section{ACLY and SREBPI Knockdown Promoted Apoptosis in Colorectal Cancer Cells}

Annexin V-APC/7- AAD and flow cytometry were used to analyze apoptosis in CRC cell lines (Figure 3A-C). The results obtained from the preliminary analysis showed an increase in the rate of apoptotic cells in the $A C L Y$ and SREBP1 knockdown cells. Further analysis showed that the proportion of apoptotic cells in the $A C L Y$ knockdown cells was lower than that in the SREBP1 knockdown cells, although the difference was not statistically significant. Our results suggested that knockdown of $A C L Y$ and SREBPI enhanced the apoptosis rate in CRC cells, with knockdown of SREBP1 playing a more potent role in promoting cell apoptosis. Next, we used a JC-1 kit to detect apoptosis (Figure 3D-F), and found that both $A C L Y$ and $S R E B P 1$ knockdown significantly increased the proportion of apoptotic Caco-2 and Lovo cells. The results of flow cytometry in the knockdown group of Caco-2 cells were slightly different from those of JC-1. Flow cytometry indicated that the apoptosis rate of $A C L Y$ knockdown cells was significantly higher than that of SREBP1 knockdown cells in Caco-2 cells.

\section{Silencing of ACLY and SREBPI Suppressed the Invasion and Migration of CRC Cells} As detected by wound healing and Transwell assays, knockdown of $A C L Y$ or SREBP1 suppressed the migration and invasion ability of CRC cells (Figure 4), in which the effect of SREBP1 knockdown was more significant. A comparison of the two sets of data revealed that SREBP1 played a more critical role in migration and invasion.

\section{ACLY and SREBPI Knockdown Inhibited the Production of Lipids and Total TGs in Cells}

High expression of SREBP1 and ACLY promotes cellular lipid synthesis. After knocking down SREBPI or ACLY expression, the distribution of lipids in Caco-2 and Lovo cells was detected using oil red $\mathrm{O}$ staining (Figure 5E). Compared with the control group cells, the results showed that knockdown of $A C L Y$ decreased the distribution area of lipid droplets in the cytoplasm, and lipids were reduced in both cytoplasm and nucleus in SREBPI knockdown cells. Similarly, the results of a total intracellular TGs assay (Figure 5B) showed that SREBPI and $A C L Y$ knockdown 
A

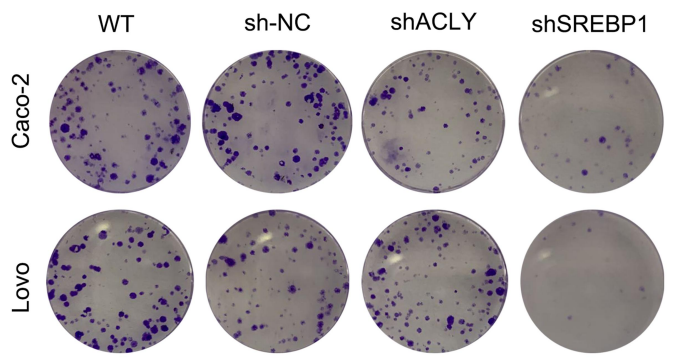

B

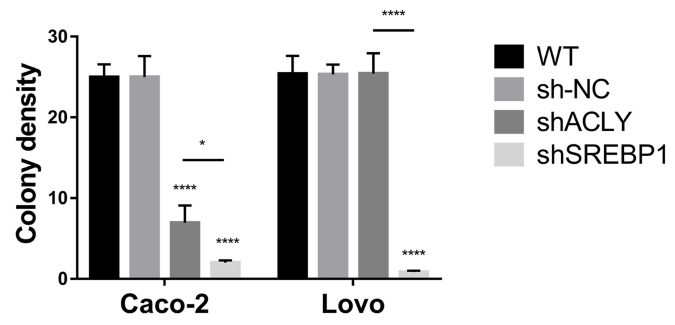

C

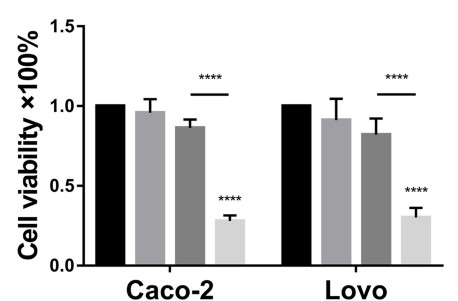

24h

D
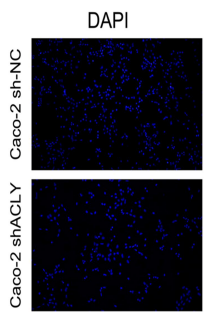

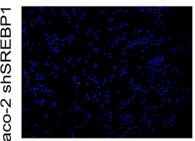
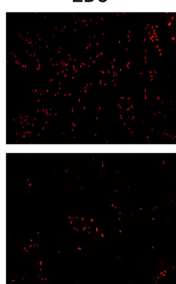

$+$

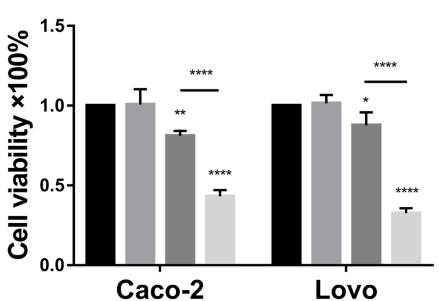

$48 \mathrm{~h}$

DAPI
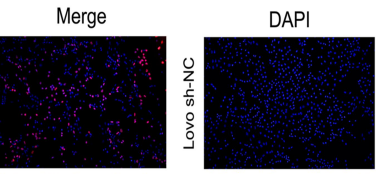

EDU
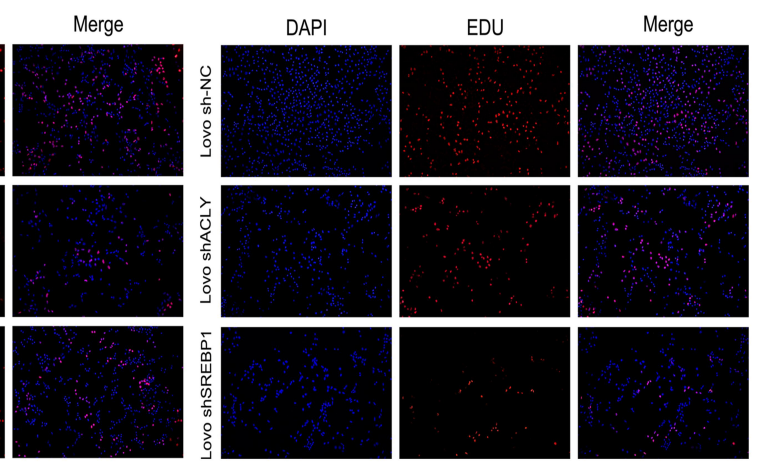

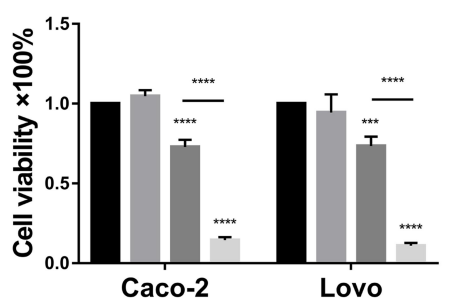

$120 \mathrm{~h}$
WT

sh-NC ShACLY ShSREBP1

Figure 2 Knockout of ACLY/SREBPI inhibited the proliferation of colorectal cancer cells. (A and B) The effect of shACLY or shSREBPI on colony formation assay in Caco-2 and Lovo cells. (C) CCK8 was used to detect the proliferation ability of cells in different groups at 24h, 48h and I20h after seeding plate. (D-F) Silencing ACLY or SREBPI decreased the replication of DNA in Caco-2 and Lovo cells. The percent of Edu-positive cells was recorded. The magnification is $40 \times$. $* P<0.05$, $* * P<0.0$, $* * * P<0.00 \mathrm{I}$, or $* * * * P<0.0001$ vs control group.

affected lipid homeostasis to varying degrees in Caco-2 and Lovo cells. Knockdown of ACLY and SREBPI reduced the content of intracellular TG in CRC cells significantly.

\section{The ACLY/SREBPI Axis Regulates Lipid Metabolism Pathways in CRC Cells}

Knockdown of $A C L Y$ or SREBP1 reduced levels of proteins related to lipid metabolism pathways, such as ACC1, LDLR, and HMGCR (Figure 5A, C and D). The ACLY level in Caco-2 following SREBP1 knockdown did not change significantly compared with that in the control group in the Western blotting results. In Caco-2 cells, $A C L Y$ knockdown decreased the level of SREBP1 significantly, while in Lovo cells, the level of SREBP1 in the
$A C L Y$ knockdown group decreased slightly. The above results suggested that the relationship between ACLY and SREBP1 in colorectal cancer cells was different from the "upstream and downstream" relationship described in most other tumors, especially in Caco-2 cells. The level of HMGCR reflects the degree of total lipid metabolism to some extent, and the HMGCR levels in both cell knockdown groups decreased significantly. LDLR is located downstream of SREBP1 and is one of the important factors regulating cholesterol metabolism. Knockdown of $A C L Y$ and SREBPI significantly decreased the level of LDLR in both cell lines. In Lovo cells, the decrease of LDLR in the $A C L Y$ knockdown group was more significant than that in the SREBP1 knockdown group. The results of most studies have shown that ACC1 is located downstream of ACLY and 
A

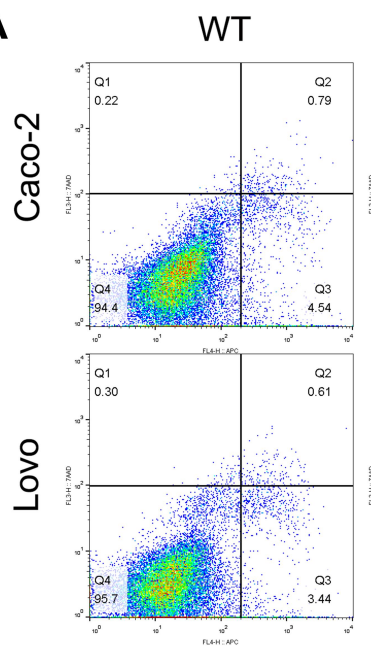

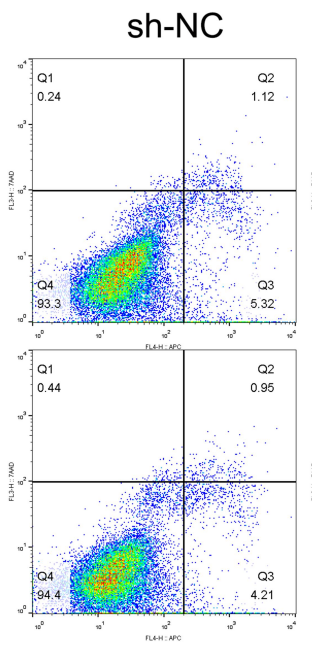

D
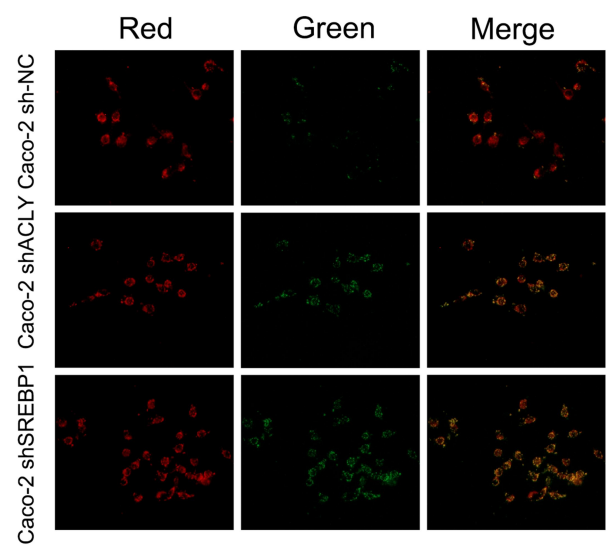
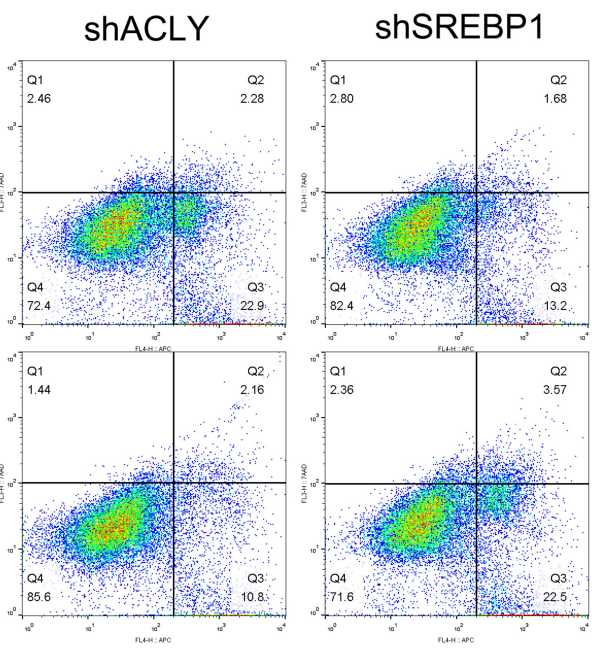

B

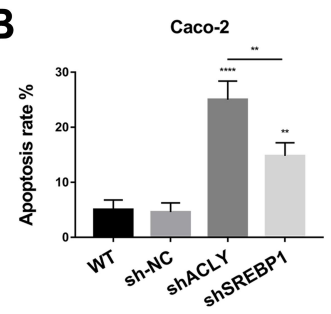

C
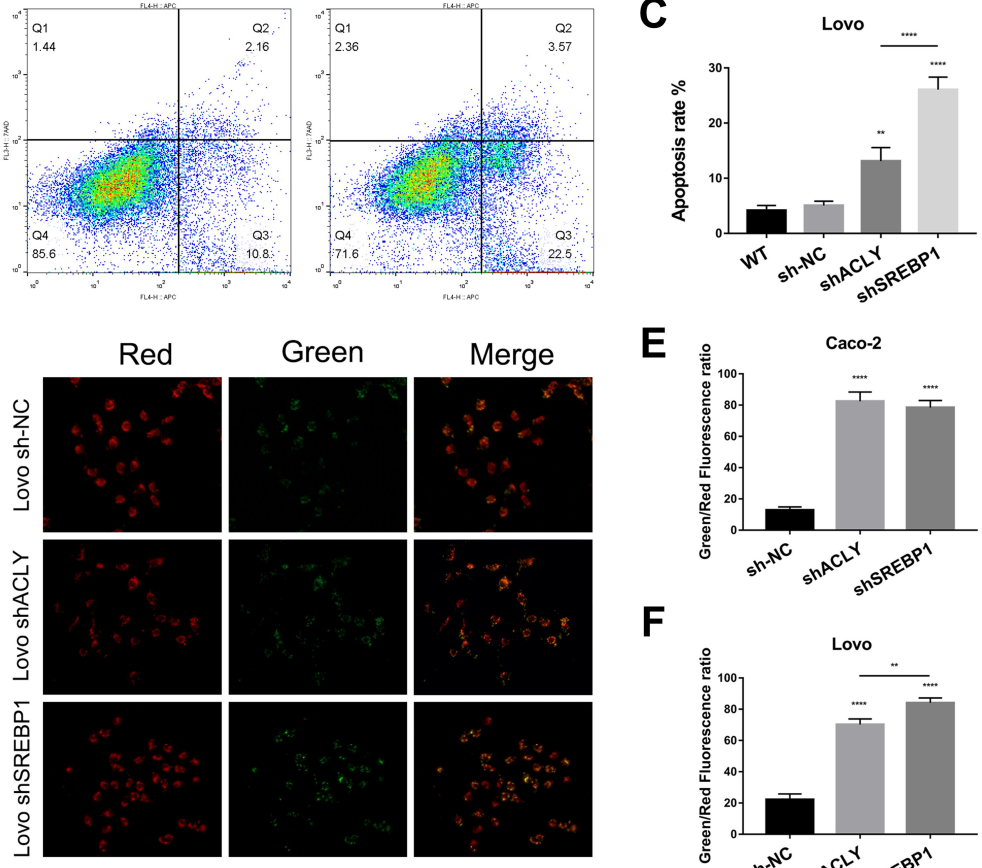

E

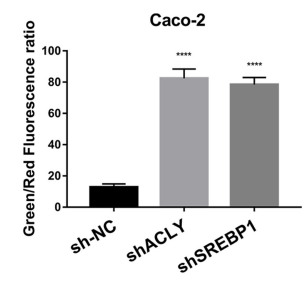

$F$

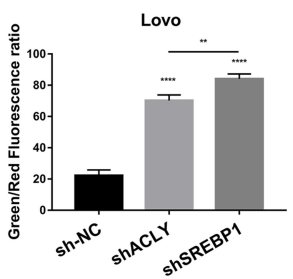

Figure 3 ACLY/SREBPI knockdown promoted apoptosis in colorectal cancer cells. (A) Caco-2 and Lovo cells were transfected with control shRNA, ACLY-siRNA or SREBPI-siRNA and subjected to an Annexin V-APC and 7AAD staining assay prior to flow cytometric analysis. Proportion of Annexin V-positive and 7AAD-positive cells post-shRNA transfection of (B) Caco-2 and (C) Lovo cells. (D-F) Results of an JC-I assay showed that the knockdown of ACLY or SREBPI in Caco-2 and Lovo cells reduced mitochondrial membrane potential. Red reflected normal membrane potential level of cells. Green fluorescence reflected the decline of mitochondrial membrane potential means apoptosis. The magnification is $200 \times$. $* * P<0.01$, or $* * * * P<0.0001$ vs control group.

is one of the key substrate enzymes that regulate DNL. ACC1 levels were decreased significantly in the $A C L Y$ and SREBP1 knockdown groups in both cell lines.

\section{Discussion}

As mentioned in the introduction, SREBP1 is an important upstream molecule of lipid metabolism, and its involvement in tumor metabolism has attracted increased research attention. ACLY has become a research hotspot of tumor lipid metabolism in recent years. DNL is the main lipid metabolism mechanism in tumors, and ACLY catalyzes the first key substrate reaction in DNL. Therefore, the present study chose ACLY and SREBP1 as the research objects.

In a pre-experiment, we detected the expression levels of ACLY and SREBP1 in four colorectal cancer cell lines
(Caco-2, Lovo, HCT116, and SW480) and one normal colorectal cell (NCM460). These cell lines are immortal cells with a strong metabolic capacity; therefore, there was no significant difference in the expression of SREBP1 among them, which was similar to the results of previous studies. ${ }^{18,19}$ ACLY was highly expressed in Caco-2, Lovo, and SW480 cell lines; however, SW480 cells are mostly used in colorectal cancer metastatic tumor models. Furthermore, HCT116 has been used in a variety of lipid metabolism models; however, the expression of SREBP1 in HCT116 is relatively low, indicating low levels of lipid metabolism. Therefore, Caco-2 and Lovo cell lines were selected for further experiments.

We knocked down $A C L Y$ and SREBPI expression in Caco-2 and Lovo cell lines using lentiviruses for subsequent experiments. The results of the CCK- 8 assay indicated that 

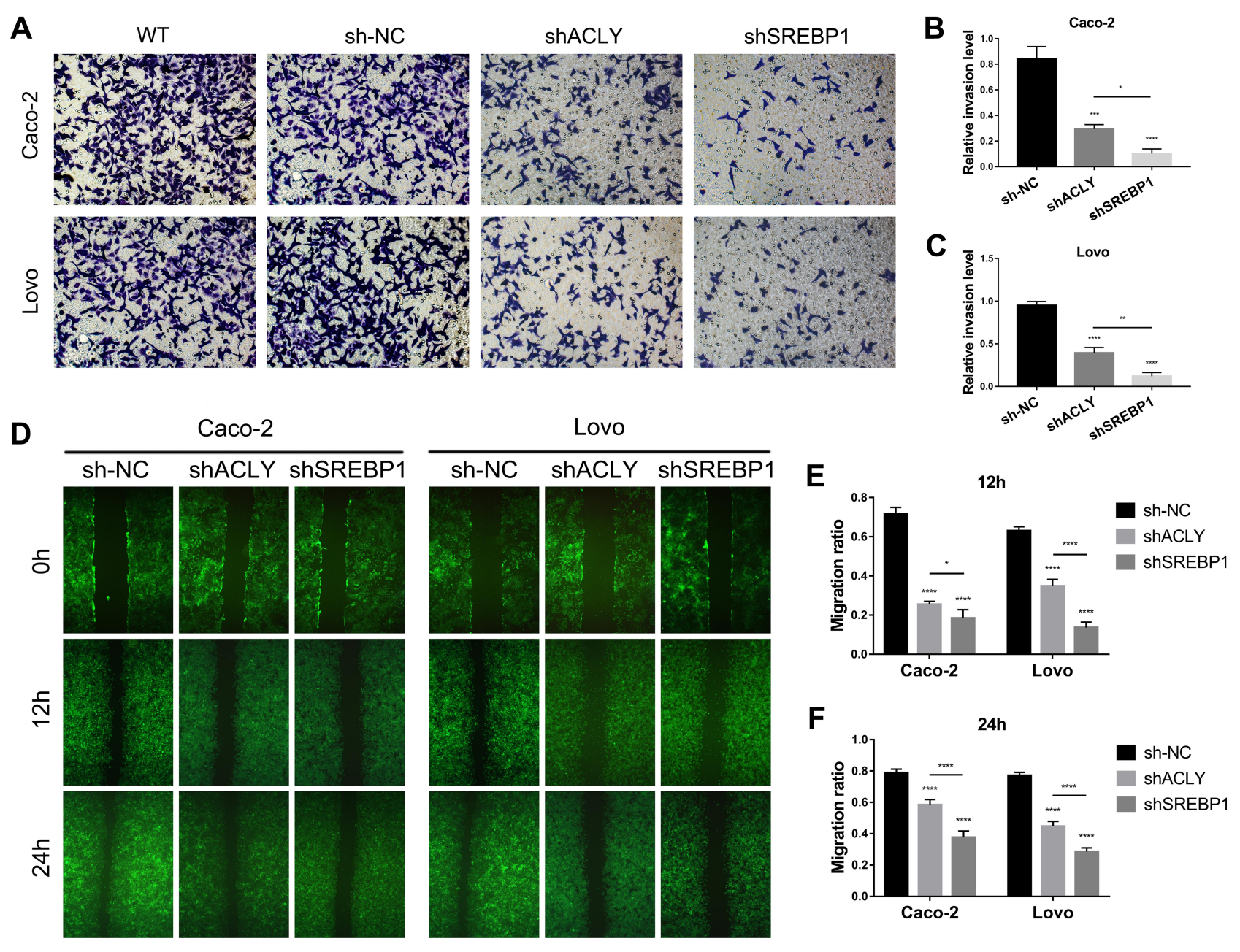

Figure 4 Silencing ACLY/SREBPI suppressed the invasion and migration of colorectal cancer cells. (A-C) The effect of shACLY or shSREBPI on transwell invasion assay in Caco-2 and Lovo cells. The magnification is $200 \times$ (D) Wound healing assay showed the ability of cell migration in each group at (E) I $2 \mathrm{~h}$ and (F) $24 \mathrm{~h}$ compared to $0 \mathrm{~h}$. The magnification is $40 \times$. $* P<0.05$, $* * P<0.01$, $* * * P<0.00$ I, or $* * * * P<0.000$ I vs control group.

knockdown SREBP1 inhibited proliferation of Caco-2 and Lovo cells, while knockdown of $A C L Y$ had little effect on tumor cell growth at $24 \mathrm{~h}$, but worked efficiently at 48 and $120 \mathrm{~h}$. We further verified that SREBP1 and ACLY facilitated the colony formation of CRC cells to varying degrees, but ACLY knockdown did not affect colony formation in Lovo cells. The above results suggested that both SREBP1 and ACLY play important roles in tumor growth in the early stage of tumor formation. However, when the tumor grew to a certain extent, the decisive effect of ACLY on tumor proliferation decreased. Other compensative pathways might exist in colorectal cancer cells after ACLY knockdown, such as the ACSS2 pathway and acetic acid metabolism pathway. ${ }^{20,21}$ Considering the EdU results, $A C L Y$ and SREBP1 knockdown had already been shown to facilitate cell proliferation at the early stage of tumor development, which is related to the degree of DNA replication, although there was no notable increase in cell counts. The results of the cell proliferation assay showed that ACLY and SREBP1 were the key proteins regulating CRC cell proliferation and both of them were closely related to the occurrence and development of CRC, in which ACLY might play a less important role in tumorigenesis.

The wound healing and Transwell invasion assay showed that knockdown of $A C L Y$ or SREBPI had obvious effects on the migration ability of colorectal cancer cells and that SREBP1 played a more important role. This evidence suggested that SREBP1 might be located upstream of ACLY in the lipid metabolism pathway in CRC, which would agree with the results of previous studies of most other tumors; however, no authoritative conclusion has been made in the study of CRC..$^{22,23}$

However, the apoptosis test yielded some different results. ACLY knockdown increased the proportion 

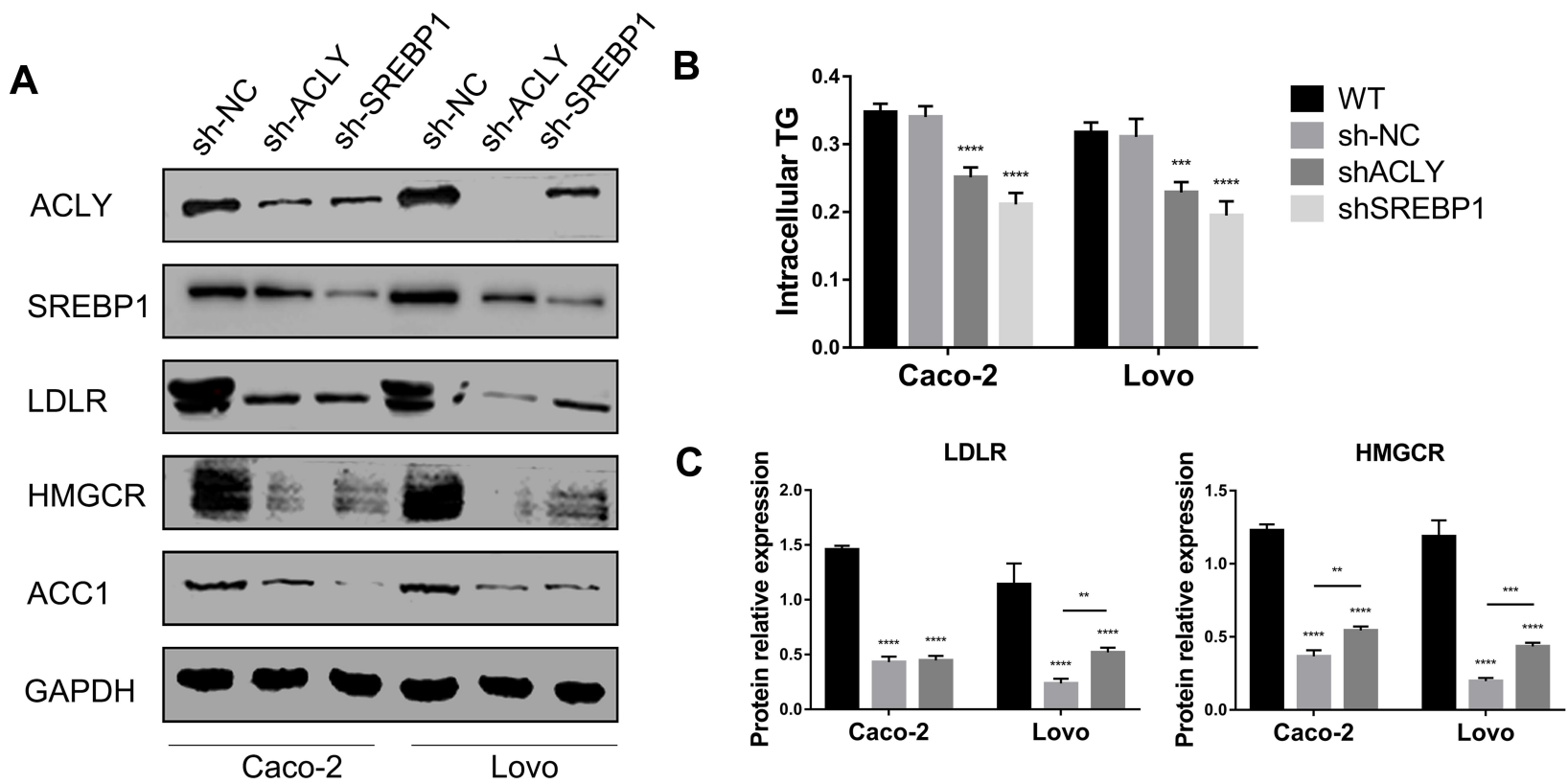

D
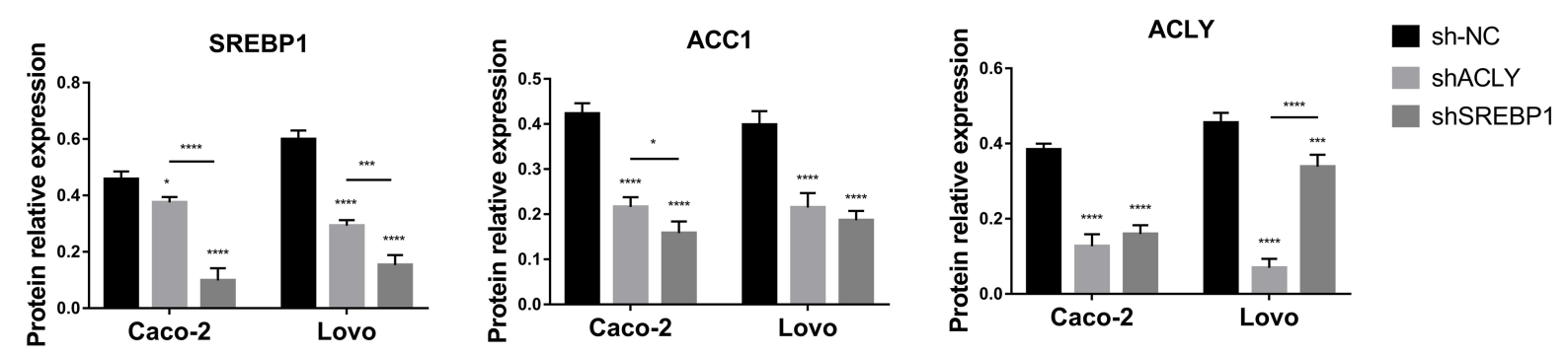

E
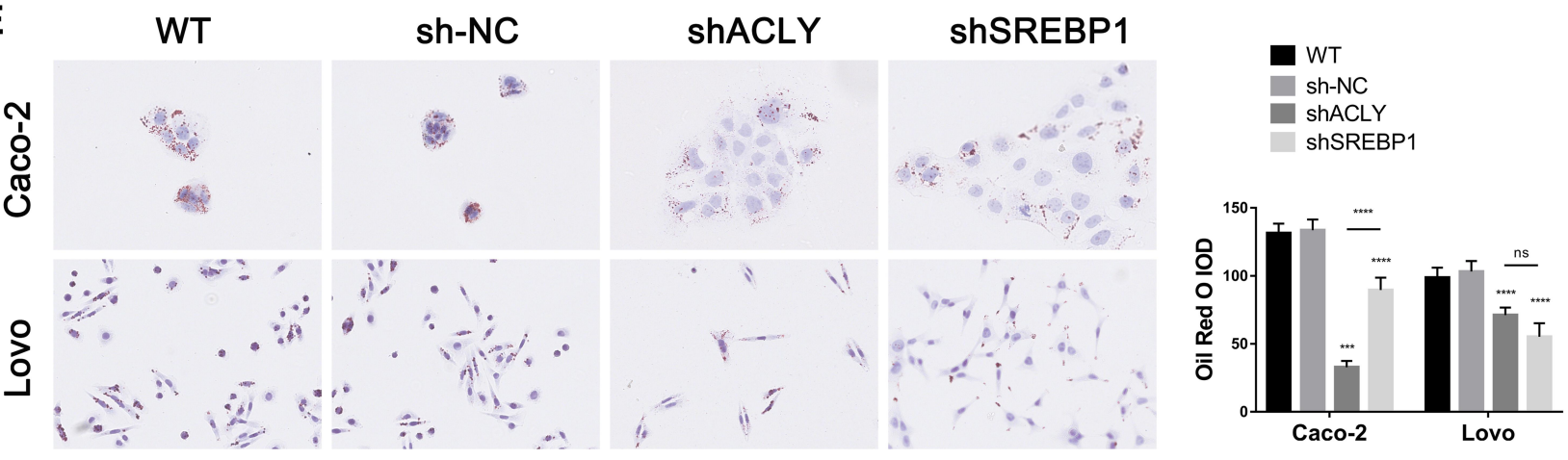

Figure 5 ACLY/SREBPI knockdown inhibited the production of lipid and total TG in cells. (B) Determination of intracellular triglycerides in each group. (A, C and D) The effects of shNC, shACLY and shSREBPI on the ACLY, SREBPI, ACCI, LDLR and HMGCR expression levels were detected by Western blotting. GAPDH was used as an internal loading control. (E) Intracellular droplets in each group on oil red staining. The magnification is $400 \times$. $* P<0.05$, $* * P<0.0$ I, $* * * P<0.00 \mathrm{I}$, ns no significant differences, or $* * * * P<0.0001$ vs control group.

apoptotic Caco-2 cells more significantly compared with SREBP1 knockdown, which was contrary to the results of Lovo cells. These results suggested that ACLY plays a more important role in the apoptotic pathway in Caco2 cells than in Lovo cells. In the JC-1 test, there was no significant difference in apoptosis between $A C L Y$ knockdown and SREBPI knockdown in Caco-2 cells, which was slightly different from the findings of flow cytometry in Caco- 2 cells. The possible interpretation might be that the cells detected by flow cytometry included late apoptotic cells and dead cells, thus the results would be more comprehensive and accurate. The results of the above two apoptosis tests indicated that SREBPI knockdown promoted the apoptosis of CRC cells to a greater extent than 
$A C L Y$ knockdown. In Caco-2 cells, $A C L Y$ knockdown led to rapid apoptosis, suggesting immediate activation of apoptosis by $A C L Y$ knockdown in Caco-2 cells, in contrast to the somewhat slower activation of apoptosis in SREBPI knockdown cells.

After knocking down $A C L Y$ or SREBP1, the levels of certain lipid metabolism_related proteins (HMGCR, LDLR, and ACC1) decreased significantly, in which HMGCR reflects total lipid metabolism, LDLR responds to cholesterol synthesis in lipid metabolism, and ACC1 corresponds to DNL. ${ }^{24,25}$ The results in most tumor cells, such as glioma, breast cancer, and prostate cancer, have shown that SREBP1 is upstream of ACLY in the tumor lipid metabolism pathway. However, our study found that when $A C L Y$ was knocked down in Lovo cells, SREBP1 levels decreased significantly, and the downregulated level of SREBP1 after ACLY knockdown was similar to the decrease in ACLY after knocking down SREBP1. More interestingly, in Caco_2 cells, SREBP1 levels decreased significantly after $A C L Y$ knockdown, while the downregulation of ACLY was not obvious after knockdown of SREBP1. The results showed that in Caco-2 cells, ACLY was likely to act upstream of SREBP1; however, this was theoretically contrary to the observed level of ACC1, the third key substrate enzyme of DNL, which shows that the relationship between SREBP1 and ACLY is more complex in these cells. $^{26,27}$

It is generally believed that SREBP1 an upstream regulatory protein in the tumor lipid metabolism pathway, and most clinical studies ${ }^{28}$ have assessed compounds that induce insulin_related genes and SREBP cleavage-activating protein, as well as affecting the synthesis of downstream cholesterol, ${ }^{29,30}$ and even targeting ACLY as the first key substrate enzyme of DNL. ${ }^{31,32}$ ACLY and SREBP1 are also key factors connecting glucose metabolism and lipid metabolism. ${ }^{33}$ The significance of SREBP1 and ACLY in tumor lipid metabolism is relatively clear, and knocking down both genes aimed to refine the study of tumor lipid metabolism and find more suitable therapeutic targets. Caco-2 is a commonly used cell model in the study of inflammatory colitis and other inflammatory diseases. ${ }^{34}$ Inflammation has been linked to the pathogenesis of multiple cancers. $^{35,36}$ This study selected this cell line as the research object because on the one hand, considering the individual differences of patients with $\mathrm{CRC}$, this novel CRC cell line can expand the therapeutic targets of mechanistic pathways; and on the other hand, this model may serve as a bridge between inflammation and tumors, allowing the exploration of the possible mechanisms of human diseases.

Based on the results of the present study, we concluded that knockdown of ACLY and SREBPI restrained CRC cells' proliferation, migration, and invasion capabilities via regulation of lipid metabolism. Overall, our data provide evidence that the ACLY/SREBP1 axis enhances the sensitivity of CRC cells to apoptosis. These findings suggest that ACLY and SREBP1 could be used as dual targets for individualized therapy of colorectal cancer, with great prospects in gene, immunity, nutritional, and metabolic therapy; however, the detailed mechanism remains to be further studied.

\section{Acknowledgments}

This work was supported by the Key Laboratory of Hubei Province for Digestive System Disease, and the Central Laboratory of Renmin Hospital of Wuhan University. The sponsors had no role in any of the stages from study design to submission of the paper for publication. The authors thank Mrs. Xiaoyi Zhang from Hospital of Wuhan University for her laboratory assistance, help, advice and article polishing.

\section{Author Contributions}

Zhendong Qiu participated in the whole process of this study, and Wenhong Deng also provided guidance in various aspects. Weixing Wang and Jia $\mathrm{Yu}$ are the main leaders of this study, supervising and guiding the research work. Yupu Hong, Liang Zhao, Man Li, Yongjun Guan, Yingru Su, Chen Chen and Qiao Shi play an important role in experimental technology and thesis writing. All authors made a significant contribution to the work reported, whether that is in the conception, study design, execution, acquisition of data, analysis and interpretation, or in all these areas; took part in drafting, revising or critically reviewing the article; gave final approval of the version to be published; have agreed on the journal to which the article has been submitted; and agree to be accountable for all aspects of the work.

\section{Disclosure}

The authors report no conflicts of interest in this work.

\section{References}

1. Castejon M, Plaza A, Martinez-Romero J, et al. Energy restriction and colorectal cancer: a call for additional research. Nutrients. 2020;12 (1):114. doi:10.3390/nu12010114 
2. Zhu J, Thompson CB. Thompson CB metabolic regulation of cell growth and proliferation. Nat Rev Mol Cell Biol. 2019;20:436-450. doi:10.1038/s41580-019-0123-5

3. Zaidi N, Swinnen JV, Smans K. ATP-citrate lyase: a key player in cancer metabolism. Cancer Res. 2012;72(15):3709-3714. doi:10.1158/0008-5472.CAN-11-4112

4. Yue B, Liu C, Sun H, et al. A positive feed-forward loop between LncRNA-CYTOR and Wnt/beta-catenin signaling promotes metastasis of colon cancer. Mol Ther. 2018;26(5):1287-1298. doi:10.1016/j. ymthe.2018.02.024

5. Du T, Sikora MJ, Levine KM, et al. Key regulators of lipid metabolism drive endocrine resistance in invasive lobular breast cancer. Breast Cancer Res. 2018;20(1):106. doi:10.1186/s13058-018-1041-8

6. Dutta A, Sharma-Walia N. Curbing lipids: impacts on cancer and viral infection. Int J Mol Sci. 2019;20(3):644. doi:10.3390/ ijms20030644

7. Damiano F, Giannotti L, Gnoni GV, et al. Quercetin inhibition of SREBPs and ChREBP expression results in reduced cholesterol and fatty acid synthesis in C6 glioma cells. Int J Biochem Cell Biol. 2019;117:105618. doi:10.1016/j.biocel.2019.105618

8. Clarke A. Energy flow in growth and production. Trends Ecol Evol. 2019;34:502-509. doi:10.1016/j.tree.2019.02.003

9. Bertolio R, Napoletano F, Mano M, et al. Sterol regulatory element binding protein 1 couples mechanical cues and lipid metabolism. Nat Commun. 2019;10(1):1326. doi:10.1038/s41467-019-09152-7

10. Vantaggiato C, Panzeri E, Citterio A, et al. Antipsychotics promote metabolic disorders disrupting cellular lipid metabolism and trafficking. Trends Endocrinol Metab. 2019;30(3):189-210. doi:10.1016/j.tem.2019.01.003

11. Cheng C, Ru P, Geng F, et al. Glucose-mediated N-glycosylation of SCAP is essential for SREBP-1 activation and tumor growth. Cancer Cell. 2015;28(5):569-581. doi:10.1016/j.ccell.2015.09.021

12. Wei J, Leit S, Kuai J, et al. An allosteric mechanism for potent inhibition of human ATP-citrate lyase. Nature. 2019;568:566-570. doi:10.1038/s41586-019-1094-6

13. Verschueren KHG, Blanchet C, Felix J, et al. Structure of ATP citrate lyase and the origin of citrate synthase in the Krebs cycle. Nature. 2019;568:571-575. doi:10.1038/s41586-019-1095-5

14. Bauer DE, Hatzivassiliou G, Zhao F, et al. ATP citrate lyase is an important component of cell growth and transformation. Oncogene. 2005;24(41):6314-6322. doi:10.1038/sj.onc.1208773

15. Li H, Sartorelli V. ATP citrate lyase: a new player linking skeletal muscle metabolism and epigenetics. Trends Endocrinol Metab. 2018;29(4):202-204. doi:10.1016/j.tem.2018.01.006

16. Zu XY, Zhang QH, Liu JH, et al. ATP citrate lyase inhibitors as novel cancer therapeutic agents. Recent Pat Anticancer Drug Discov. 2012;7(2):154-167. doi:10.2174/157489212799972954

17. Bouchard-Mercier A, Rudkowska I, Lemieux S, et al. Polymorphisms, de novo lipogenesis, and plasma triglyceride response following fish oil supplementation. J Lipid Res. 2013;54 (10):2866-2873. doi:10.1194/jlr.M041590

18. Cheng Y, Jia B, Wang Y, et al. miR-133b acts as a tumor suppressor and negatively regulates ATP citrate lyase via PPARgamma in gastric cancer. Oncol Rep. 2017;38(5):3220-3226. doi:10.3892/or.2017.5944

19. Csanadi A, Kayser C, Donauer M, et al. Prognostic value of malic enzyme and ATP-citrate lyase in non-small cell lung cancer of the young and the elderly. PLoS One. 2015;10(5):e0126357. doi:10.1371/ journal.pone. 0126357

20. Gao X, Lin SH, Ren F, et al. Acetate functions as an epigenetic metabolite to promote lipid synthesis under hypoxia. Nat Commun. 2016;7:11960. doi:10.1038/ncomms 11960
21. Comerford SA, Huang Z, Du X, et al. Acetate dependence of tumors. Cell. 2014;159(7):1591-1602. doi:10.1016/j.cell.2014.11.020

22. Ippolito L, Morandi A, Giannoni E, et al. Lactate: a metabolic driver in the tumour landscape. Trends Biochem Sci. 2019;44(2):153-166. doi:10.1016/j.tibs.2018.10.011

23. Zhang C, Liu J, Huang G, et al. Cullin3-KLHL25 ubiquitin ligase targets ACLY for degradation to inhibit lipid synthesis and tumor progression. Genes Dev. 2016;30(17):1956-1970. doi:10.1101/ gad.283283.116

24. Wang C, Tong Y, Wen Y, et al. Hepatocellular carcinoma-associated protein TD26 interacts and enhances sterol regulatory element-binding protein 1 activity to promote tumor cell proliferation and growth. Hepatology. 2018;68(5):1833-1850. doi:10.1002/ hep. 30030

25. Zhou J, Qu G, Zhang G, et al. Glycerol kinase 5 confers gefitinib resistance through SREBP1/SCD1 signaling pathway. J Exp Clin Cancer Res. 2019;38(1):96. doi:10.1186/s13046-019-1057-7

26. Lin YC, Wu MS, Lin YF, et al. Nifedipine modulates renal lipogenesis via the AMPK-SREBP transcriptional pathway. Int J Mol Sci. 2019;20(7).

27. Zhang $\mathrm{Y}, \mathrm{Li} \mathrm{C}, \mathrm{Hu} \mathrm{C}$, et al. Lin28 enhances de novo fatty acid synthesis to promote cancer progression via SREBP-1. EMBO Rep. 2019;20(10):e48115. doi:10.15252/embr.201948115

28. Xiang AS, Kingwell BA. Rethinking good cholesterol: a clinicians' guide to understanding HDL. Lancet Diabetes Endocrinol. 2019;7:575-582. doi:10.1016/S2213-8587(19)30003-8

29. Syafruddin SE, Rodrigues P, Vojtasova E, et al. A KLF6-driven transcriptional network links lipid homeostasis and tumour growth in renal carcinoma. Nat Commun. 2019;10(1):1152. doi:10.1038/ s41467-019-09116-X

30. Shi Z, Zhou Q, Gao S, et al. Silibinin inhibits endometrial carcinoma via blocking pathways of STAT3 activation and SREBP1-mediated lipid accumulation. Life Sci. 2019;217:70-80. doi:10.1016/j. 1fs.2018.11.037

31. Hatzivassiliou G, Zhao F, Bauer DE, et al. ATP citrate lyase inhibition can suppress tumor cell growth. Cancer Cell. 2005;8 (4):311-321. doi:10.1016/j.ccr.2005.09.008

32. Wang G, Li Z, Li X, et al. RASAL1 induces to downregulate the SCD1, leading to suppression of cell proliferation in colon cancer via LXRalpha/SREBP1c pathway. Biol Res. 2019;52(1):60. doi:10.1186/ s40659-019-0268-X

33. Xu D, Wang Z, Xia Y, et al. The gluconeogenic enzyme PCK1 phosphorylates INSIG1/2 for lipogenesis. Nature. 2020;580 (7804):530-535. doi:10.1038/s41586-020-2183-2

34. Chiriac MT, Buchen B, Wandersee A, et al. Activation of epithelial signal transducer and activator of transcription 1 by interleukin 28 controls mucosal healing in mice with colitis and is increased in mucosa of patients with inflammatory bowel disease. Gastroenterology. 2017;153(1):123-138 e8. doi:10.1053/j. gastro.2017.03.015

35. Li N, Zhou ZS, Shen Y, et al. Inhibition of the sterol regulatory element-binding protein pathway suppresses hepatocellular carcinoma by repressing inflammation in mice. Hepatology. 2017;65 (6):1936-1947. doi:10.1002/hep.29018

36. Guo Y, Wu R, Gaspar JM, et al. DNA methylome and transcriptome alterations and cancer prevention by curcumin in colitis-accelerated colon cancer in mice. Carcinogenesis. 2018;39(5):669-680. doi:10.1093/carcin/bgy043 


\section{Publish your work in this journal}

OncoTargets and Therapy is an international, peer-reviewed, open access journal focusing on the pathological basis of all cancers, potential targets for therapy and treatment protocols employed to improve the management of cancer patients. The journal also focuses on the impact of management programs and new therapeutic agents and protocols on patient perspectives such as quality of life, adherence and satisfaction. The manuscript management system is completely online and includes a very quick and fair peer-review system, which is all easy to use. Visit http://www.dovepress.com testimonials.php to read real quotes from published authors. 